\title{
Mixed-Valency Signature in Vibrational Inelastic Electron Tunneling Spectroscopy
}

\author{
Maite Alducin, ${ }^{1,2}$ Daniel Sánchez-Portal, ${ }^{1,2}$ Andrés Arnau, ${ }^{3,1,2}$ and Nicolás Lorente ${ }^{4}$ \\ ${ }^{1}$ Centro de Física de Materiales Centro Mixto CSIC-UPV/EHU, Edificio Korta, 20018 San Sebastián, Spain \\ ${ }^{2}$ Donostia International Physics Center DIPC, P. Manuel de Lardizabal 4, 20018 San Sebastián, Spain \\ ${ }^{3}$ Departamento de Física de Materiales, Facultad de Química, UPV/EHU, 20080 San Sebastián, Spain \\ ${ }^{4}$ Centre d'Investigació en Nanociència i Nanotecnologia (CSIC-ICN), E-08193 Bellaterra, Spain
}

(Received 25 January 2010; published 31 March 2010)

\begin{abstract}
Density functional theory simulations of the vibrational inelastic electron tunneling spectroscopy (IETS) of $\mathrm{O}_{2}$ on $\mathrm{Ag}(110)$ permits us to solve its unexplained IETS data [Hahn et al., Phys. Rev. Lett. $\mathbf{8 5}, 1914(2000)]$. When semilocal density functional theory is corrected by including static intra-atomic correlations, the IETS simulations are in excellent agreement with the experiment. The unforeseen consequence of our calculations is that when adsorbed along the [001] direction, molecular $\mathrm{O}_{2}$ on $\mathrm{Ag}(110)$ is a mixed-valent system. This analysis of IETS unambiguously reveals the paramagnetic nature of $\mathrm{O}_{2}$ on $\operatorname{Ag}(110)$.
\end{abstract}

DOI: 10.1103/PhysRevLett.104.136101

PACS numbers: 68.37.Ef, 72.10.-d, 72.25.-b, 79.20.Rf

Inelastic electron tunneling spectroscopy (IETS) performed with the scanning tunneling microscope (STM) has given us unprecedented insight into the chemical structure of matter on the atomic scale [1-3]. In this respect, state-of-the-art theoretical simulations, which use the electronic structure calculated with local or semilocal density functional theory (DFT) as the initial ingredient, have proven to be essential to interpret the recorded STM [4] and the IETS signals [5-7]. Still, there is an emblematic experiment for which most sophisticated IETS simulations have failed: the conductance decreases observed over the vibrational thresholds of $\mathrm{O}_{2}$ on $\mathrm{Ag}(110)$ [8]. More precisely, STM-IETS gives signals for only one of the chemisorption states, the one with the molecular axis along the [001] surface direction (henceforth, $\mathrm{O}_{2}[001]$ ).

In general, the IETS signal corresponds to an increase of the STM conductance because the excitation of a vibrational mode opens an additional channel for conduction [5,9-11]. Nevertheless, when a molecular state is resonant with the Fermi energy $\left(E_{F}\right)$, virtual vibrational excitations are important, and a decrease in conductance is predicted $[9,10]$. To date, the $\mathrm{O}_{2}[001]$ is the only case for which conductance decreases have been reported. However, the simulated IETS of $\mathrm{O}_{2}[001]$ have consistently produced increases of conductance instead of the experimental decreases [7,10]. Given that the DFT electronic structure predicts $\pi_{g}$ resonances away from $E_{F}$ [see Fig. 1(a)], the published conclusions were that the $\pi_{g}$ resonances were wrongly positioned with respect to the Fermi level $[7,10]$. Nevertheless, questioning the position of the Fermi level and, thus, the molecular charge state in this system is at variance with the excellent characterization of the two experimental chemisorption states given by this theory [12], the $\mathrm{O}_{2}[001]$, and the one with the molecular axis along the $[1 \overline{1} 0]$ direction $\left(\mathrm{O}_{2}[1 \overline{1} 0]\right)$. Moreover, the Fermi level fitting cannot reproduce the rich IETS structure of the experimental data showing increases and decreases according to the vibrational mode and the tip localization.

In this Letter, we present DFT calculations corrected by static correlations $(\mathrm{DFT}+U)$ [13] in combination with STM-IETS simulations [5,7]. We find that the chemisorbed $\mathrm{O}_{2}$ retains a finite spin moment only in one of the two possible chemisorption geometries, namely, the $\mathrm{O}_{2}[001]$. The origin of this paramagnetic state is a molecular orbital pinned at the Fermi level, giving rise to a molecular mixedvalent state [14]. The corresponding electronic structure leads to IETS simulations where the experimental change in conductance and also their spatial tip-position dependence are explained giving unprecedented information on the electronic and vibrational symmetries at work.

Density functional theory calculations are performed with the VASP code using plane waves with an energy cutoff

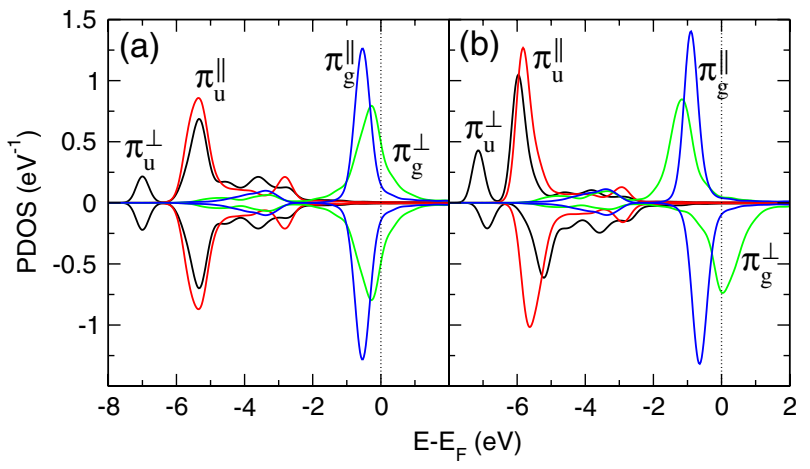

FIG. 1 (color online). Spin-up (positive) and spin-down (negative) projected density of states of $\mathrm{O}_{2} / \mathrm{Ag}(110)$ onto the $\mathrm{O}_{2}$ molecular orbitals. The molecule is chemisorbed along the [001] direction. (a) Semilocal DFT (GGA) results. (b) Semilocal DFT $+U$ results $(U=3 \mathrm{eV})$. Intra-atomic correlation leads to a change in the $\pi_{g}$ resonance by splitting its spin components following a Stoner-like process. The Gaussian broadening used in the projection is $0.25 \mathrm{eV}$. 
TABLE I. Semilocal DFT (GGA) and DFT $+U(\mathrm{GGA}+U)$ quantities describing the physical properties of $\mathrm{O}_{2}$ chemisorbed on $\mathrm{Ag}(110)$ along the [001] direction.

\begin{tabular}{lccccc}
\hline \hline & $\begin{array}{c}\mathrm{O}-\mathrm{O} \\
(\AA)\end{array}$ & $\begin{array}{c}\mathrm{O}-\mathrm{Ag} \\
(\AA)\end{array}$ & $\begin{array}{c}\mathrm{O}_{2} \text {-surface } \\
(\AA)\end{array}$ & $\begin{array}{c}\mathrm{O}_{2} \text {-induced charge } \\
(\text { Bader analysis in } e)\end{array}$ & $\begin{array}{c}\text { magnetization } \\
\left(\mu_{B}\right)\end{array}$ \\
\hline $\mathrm{GGA}$ & 1.43 & 2.35 & 1.34 & 0.95 & 0.0 \\
$\mathrm{GGA}+U=2 \mathrm{eV}$ & 1.42 & 2.36 & 1.34 & 0.93 & 0.38 \\
$\mathrm{GGA}+U=3 \mathrm{eV}$ & 1.41 & 2.37 & 1.34 & 0.88 & 0.54 \\
$\mathrm{GGA}+U=4 \mathrm{eV}$ & 1.39 & 2.39 & 1.34 & 0.83 & 0.68 \\
\hline \hline
\end{tabular}

of $515 \mathrm{eV}$ and the projector augmented wave method (PAW) [15]. We use a periodic supercell consisting of a six-layer slab separated by $10.45 \AA$ of vacuum and a large $(3 \times 4)$ surface unit cell in order to approach the dilute overlayer limit. The surface Brillouin zone is sampled with a $4 \times 4 \times 1$ Monkhorst-Pack grid of special $k$ points. The exchange and correlation energy is calculated within the generalized gradient approximation (GGA) using the Perdew and Wang functional (PW91) [16]. Vibrational modes are then obtained from the diagonalization of the dynamical matrix, which is calculated by a finitedifference method implemented in VASP. The IETS simulations are performed using the many-body extension of the Tersoff-Hamman approximation for the STM [5,7].

The inclusion of static correlations via the DFT $+U$ treatment does not impoverish the excellent GGA description of the adsorption molecular properties. Indeed, the DFT $+U$ method is known to correct the electronic spectrum without perturbing the physical magnitudes for which the standard DFT excels. A detailed comparison of these quantities is shown in Table I. Corrections to the screened on-site Coulomb repulsion are applied to the oxygen $2 p$ electrons [17] following the rotationally invariant DFT + $U$ scheme proposed by Dudarev et al. [18]. The electronic structure giving rise to the mixed-valent regime, Fig. 1(b), is obtained for $2 \mathrm{eV} \leq U \leq 4 \mathrm{eV}$. The computed $U$ is about $4 \mathrm{eV}$ using the constrained DFT of Cococcioni and Gironcoli [19] to evaluate the energy cost of adding extra charge to $\mathrm{O}_{2}$ when adsorbed on $\operatorname{Ag}(110)$. The $U$ value depends on the actual projectors and the constraining method used to fix the orbital occupation. However, we have verified that such limitation does not affect the final electronic structure in the present case.

The molecular electronic structure is subject to a Stonerlike process for the chemisorbed $\mathrm{O}_{2}$ [001], but not for the $\mathrm{O}_{2}[1 \overline{1} 0]$. The large screening of the molecule chemisorbed along the $[1 \overline{1} 0]$ direction quenches its intrinsic magnetism. Along the [001] axis, the molecule is more distant from the surface leading to a reduced screening and a stronger molecular character. This is seen in Fig. 1. There, the density of states (DOS) is plotted according to its molecular character by projecting it onto the $\mathrm{O}_{2}$ molecular orbitals (MO). As seen in Fig. 1(b), there is a Stoner-like splitting of the highest occupied MO due to the intramolecular $U$. The spin splitting of this orbital, the perpendicular-to-the- surface $\pi_{g}$ (henceforth, $\pi_{g}^{\perp}$ ), entails two fundamental consequences: (i) the survival of a molecular magnetic moment and (ii) the pinning of the minority spin $\pi_{g}^{\perp}$ to the Fermi level. These results add new physics to the understanding of this long-standing puzzle: the $\mathrm{O}_{2} / \mathrm{Ag}(110)$ along the [001] direction is a mixed-valency system.

Our simulated results are indeed in excellent agreement with the experiments. Not only is the spatial distribution reproduced, but the actual values of the conductance change $\Delta \sigma / \sigma$, where $\sigma=d I / d V$, follow the experimental trends [20]. Figure 2 summarizes the main results of our simulations and allows the direct comparison with the experiment (Fig. 4 of Hanh et al. [8]). First, we observe that the constant current image simulation of Figs. 2(a) and 2(b) reproduces the main features of the topographic image recorded in the experiments. The large DOS of $\pi_{g}^{\perp}$ character that is observed in Fig. 1(b) around the Fermi energy is viewed as two protrusions along the molecular axis in the STM image. Our STM simulation is also quite similar to the results of Olsson et al. [21], who performed spinunpolarized DFT calculations of the system. Such an agreement clearly shows that information about the spin moment cannot be directly extracted from the STM topography. In fact, the mixed-valent character of $\mathrm{O}_{2}$ [001] is ultimately confirmed by the agreement reached with the experimental IETS for which the standard DFT simulation fails. Figures 2(c) and 2(d) show the spatial distribution of the changes in conductance for the $\mathrm{O}-\mathrm{O}$ stretch mode. In agreement with experiment, the overall change in conductance is always negative, except on the center of the molecule that is basically zero. The simulation for the antisymmetric stretch mode is shown in Figs. 2(e) and 2(f). Here, the change in conductance over the $\mathrm{O}$ atoms is negative, but it becomes positive in the region between them, showing that our simulations capture the complex behavior observed in the experiment.

In order to understand the above results, Fig. 3 shows for each mode the conductance change separated in its inelastic contribution $\left(\Delta \sigma_{\text {ine }} / \sigma\right)$ due to the opening of a new channel, hence positive, and the renormalization of the elastic channel $\left(\Delta \sigma_{\text {ela }} / \sigma\right)$ due to the presence of the vibration that leads to a negative contribution $[9,22]$. The main difference between them despite their sign is that the inelastic contribution involves all possible intermediate electronic states, while the states contributing to the elastic 

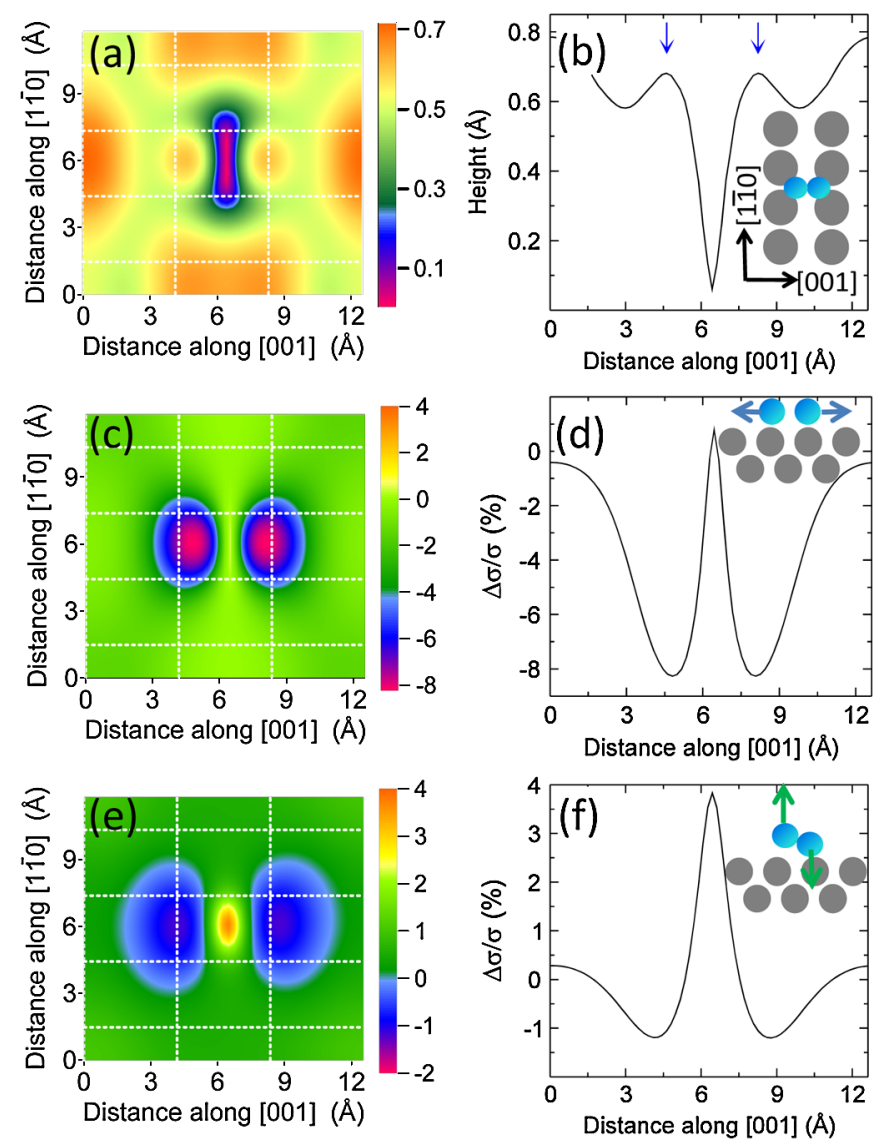

FIG. 2 (color online). Calculated GGA $+U$ local DOS and STM-IETS for $\mathrm{O}_{2}$ chemisorbed on $\mathrm{Ag}(110)$ along the [001] direction [see inset of (b)]. Results for $U=3 \mathrm{eV}$. (a) Topographic image and (b) profile along the molecular axis (both in $\AA$ ) calculated with a sample bias $V=200 \mathrm{mV}$. The zero height corresponds to a tip-surface distance of $\sim 6 \AA$. The protrusions attributed to the $\pi_{g}^{\perp}$ orbital are indicated by arrows. Calculated spatial distribution of the conductance change percentages $\Delta \sigma / \sigma(\%)$ for (c) the $\mathrm{O}-\mathrm{O}$ stretch mode $(V=200 \mathrm{mV})$ and (e) the antisymmetric stretch mode $(V=150 \mathrm{mV})$. (d) and (f) Cross sections of (c) and (e), respectively, along the molecular axis. Note the negative and positive conductance changes observed on the antisymmetric mode, in contrast to the overall negative values of the $\mathrm{O}-\mathrm{O}$ stretch mode. The grid lines in (a), (c), and (e) show the surface structure.

channel are restricted to an energy interval of the order of the mode frequency $\hbar \Omega$ about the Fermi energy [5]. The topography of the conductance change observed on the $\mathrm{O}_{2}$ [001] is determined by all the electronic states coupling with the $\pi_{g}^{\perp}$ orbital, which is the state at $E_{F}$ controlling the electronic transport process [see Fig. 1(b)]. The symmetry analysis summarized in the table of Fig. 3(e) provides a preliminary idea of the states that couple with the $\pi_{g}^{\perp}$ for each vibrational mode. In case of having various symmetry-allowed couplings, the IETS image is mainly formed by those orbitals with a larger DOS around $E_{F}$ [23]. The symmetry characters are analyzed with respect to two elements of symmetry of the system's point group, namely, reflection by the perpendicular-to-the-surface planes con-
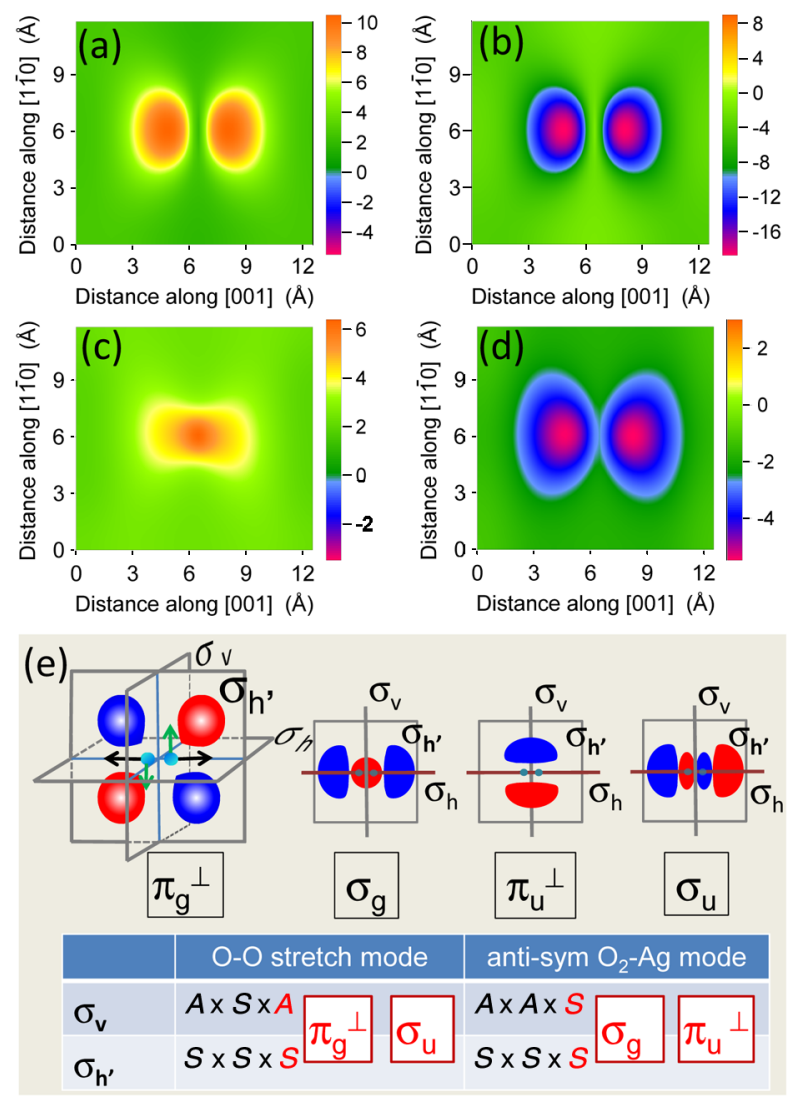

FIG. 3 (color online). Inelastic $\left(\Delta \sigma_{\text {ine }} / \sigma\right)$ and elastic contributions $\left(\Delta \sigma_{\text {ela }} / \sigma\right)$ to the conductance changes of Fig. 2 (in percentage). (a) Inelastic and (b) elastic contributions for the $\mathrm{O}-\mathrm{O}$ stretch mode. (c) Inelastic and (d) elastic for the antisymmetric stretch mode. (e) Sketch and symmetries of the MOs and modes involved in the IETS images. The Table summarizes the MOs that can couple with the $\pi_{g}^{\perp}$ to assure a nonzero matrix element $\left\langle\psi_{m, \mathbf{k}}|v| \psi_{n, \mathbf{k}}\right\rangle$ (see Ref. [6]). The symmetric $(S)$ or antisymmetric $(A)$ character of the $\pi_{g}^{\perp}$ and the electron-vibration coupling $v$ is written in black following this order for each vibrational mode and symmetry plane. In gray (red), the symmetry character of $\psi_{m, \mathbf{k}}$ necessary for a nonzero coupling. The MOs fulfilling those symmetry conditions are written inside the gray (red) squares.

taining the molecular axis $\sigma_{h^{\prime}}$ and perpendicular to the molecular axis $\sigma_{v}$.

Figures 3(a) and 3(b) are the inelastic and elastic contributions to the stretch mode, respectively. As written in Fig. 3(e), the $\pi_{g}^{\perp}$ is antisymmetric $(A)$ with respect to $\sigma_{v}$ and symmetric $(S)$ with respect to $\sigma_{h^{\prime}}$. Since the stretch mode is symmetric with respect to both elements, forcedly the symmetry character of the coupling electronic states has to be $A$ and $S$, respectively. This corresponds to the $\pi_{g}^{\perp}$ and $3 \sigma_{u}$ orbitals. The $\pi_{g}^{\perp}$ character dominates the DOS at $E_{F}$, and, as a consequence, both the inelastic and elastic components in Figs. 3(a) and 3(b) mostly reflect this symmetry. As the elastic correction is much larger than the inelastic one, the excitation of the stretch mode leads to a reduction in conduction except between the $\mathrm{O}$ atoms where the $\pi_{g}$ orbital presents a nodal plane. 
The antisymmetric $\mathrm{O}_{2}-\mathrm{Ag}$ stretch mode is more complex. The symmetry analysis of Fig. 3(e) shows that the $\pi_{g}^{\perp}$ can couple with the $3 \sigma_{g}$ and the perpendicular-to-thesurface $\pi_{u}$ orbitals, whose DOS about $E_{F}$ are small [see Fig. 1(b)]. Thus, for equivalent coupling strengths, we would expect a smaller conductance change for the antisymmetric $\mathrm{O}_{2}-\mathrm{Ag}$ stretch mode than for the $\mathrm{O}-\mathrm{O}$ stretch mode, as is the case. Figure 3(d) shows that the elastic contribution, which is restricted to states around $E_{F}$, contains a nodal plane between the $\mathrm{O}$ atoms and corresponds to the $\pi_{g}^{\perp}$ orbital, which dominates the DOS at $E_{F}$. In contrast, the summation over all possible intermediate states leads to both the $3 \sigma_{g}$ and the $\pi_{u}^{\perp}$ characters of the inelastic contribution, as seen in Fig. 3(c). Summing both contributions, we obtain that the total conductance change is positive in between the two oxygen atoms, where the elastic contribution is zero and the inelastic one is maximal, and negative in the region where the elastic part is maximal and the inelastic part minimal. This result shows the multilevel character of the inelastic process. In order to understand the IETS data, one needs to go beyond singlelevel models [9]. Yet, our analysis shows the overwhelming importance of having the spin-polarized $\pi_{g}^{\perp}$ orbital at the Fermi energy as a key ingredient in the $\mathrm{O}_{2} / \mathrm{Ag}(110)$ IETS.

This particular electronic structure, with a spinpolarized molecular orbital at the Fermi energy, is also responsible for the mixed-valent character of the chemisorbed $\mathrm{O}_{2}[001]$. Spin polarization naturally appears when the intramolecular correlation $U$ is larger than the resonance width $\Gamma$. Typically, $U$ is inversely proportional to the size of the molecular orbital. Hence, small and weakly adsorbed molecules presenting negative IETS are prone to be in a mixed-valency regime, as demonstrated for the $\mathrm{O}_{2}[001]$.

To summarize, we have applied the DFT $+U$ method to adsorbed $\mathrm{O}_{2}$ on $\mathrm{Ag}(110)$, and have reproduced the experimental IETS data by Hahn et al. [8]. In doing so, the chemisorption state $\mathrm{O}_{2}[001]$ is characterized as a mixedvalent state, where a spin-polarized molecular orbital dominates the electronic structure at the Fermi energy. Our calculations show that IETS can reveal important information about the electronic structure and magnetic nature of chemisorbed molecules. Thus, IETS arises as a promising tool to characterize the otherwise elusive molecular mixed-valency regime. In this regime, electron correlations are responsible of large charge fluctuations leading to nonadiabatic effects [24]. In this respect, our findings are relevant to explain recent experimental results [25] where $\mathrm{O}_{2}$ can be dissociated with controlled STM pulses when adsorbed in the [110] geometry but not in the [001] one. We suggest that while the $\mathrm{O}_{2}[001]$ is a mixedvalency system, with strong many-body correlations that imply fast vibrational damping, the $\mathrm{O}_{2}[1 \overline{1} 0]$ is not subjected to correlations and its vibrational damping is qualitatively smaller allowing dissociation.
We acknowledge financial support from the Spanish MICINN (No. FIS2007-066711-CO2-00 and No. FIS2009-12721-C04-01), and the Basque Government-UPV/EHU (Grant No. IT-366-07). Computational resources were provided by the Centre de Calcul de Midi-Pyrénées, the DIPC, and the SGI/IZOSGIker.

[1] B. C. Stipe, M. A. Rezai, and W. Ho, Science 280, 1732 (1998).

[2] W. Ho, J. Chem. Phys. 117, 11033 (2002).

[3] T. Komeda, Prog. Surf. Sci. 78, 41 (2005).

[4] W. A. Höfer, A.S. Foster, and A. L. Shluger, Rev. Mod. Phys. 75, 1287 (2003).

[5] N. Lorente and M. Persson, Phys. Rev. Lett. 85, 2997 (2000).

[6] N. Lorente et al., Phys. Rev. Lett. 86, 2593 (2001).

[7] N. Lorente, Appl. Phys. A 78, 799 (2004).

[8] J. R. Hahn, H. J. Lee, and W. Ho, Phys. Rev. Lett. 85, 1914 (2000).

[9] B. N. J. Persson and A. Baratoff, Phys. Rev. Lett. 59, 339 (1987).

[10] M. Paulsson et al., Phys. Rev. Lett. 100, 226604 (2008).

[11] O. Tal et al., Phys. Rev. Lett. 100, 196804 (2008).

[12] P. A. Gravil, D. M. Bird, and J. A. White, Phys. Rev. Lett. 77, 3933 (1996).

[13] V.I. Anisimov, F. Aryasetiaswan, and A. I. Lichtenstein, J. Phys. Condens. Matter 9, 767 (1997).

[14] A. C. Hewson, The Kondo Problem to Heavy Fermions (Cambridge University Press, Cambridge, 1993).

[15] G. Kresse and J. Furthmüller, Phys. Rev. B 54, 11169 (1996).

[16] J. P. Perdew et al., Phys. Rev. B 46, 6671 (1992).

[17] For checking purposes, we perform DFT $+U$ calculations using the SIESTA code to apply on-site Coulomb corrections to the $\mathrm{O}_{2} \pi_{g}$ electrons. There are no significant differences in the electronic structure when $U$ is applied to either the atomic $2 p$ or the molecular $\pi_{g}$ electrons.

[18] S. L. Dudarev et al., Phys. Rev. B 57, 1505 (1998).

[19] M. Cococcioni and S. de Gironcoli, Phys. Rev. B 71, 035105 (2005).

[20] The magnitude measured in the experiments is the second derivative of the tunneling current with respect to the sample bias. From these data, the conductance change over the vibrational threshold is then calculated by measuring the area of the peaks in the second derivative.

[21] F. E. Olsson, N. Lorente, and M. Persson, Surf. Sci. 522, L27 (2003).

[22] S. Monturet and N. Lorente, Phys. Rev. B 78, 035445 (2008).

[23] The weight of the couplings in $\Delta \sigma_{\text {ine }} / \sigma$ is inversely proportional to the energy difference between the $\pi_{g}^{\perp}$ and the other coupling orbital [6]. Since the $\pi_{g}^{\perp}$ orbital is at resonance with $E_{F}$, the contribution of the coupling orbitals with larger DOS at $E_{F}$ is favored.

[24] T. Brunner and D. C. Langreth, Phys. Rev. B 55, 2578 (1997).

[25] J. R. Hahn and W. Ho, J. Chem. Phys. 122, 244704 (2005). 